\title{
Propuesta de portafolio de servicios y actividades para la construcción de la paz desde las Bibliotecas de la Universidad de La Salle (Bogotá, Colombia)
}

\author{
Martha Patricia Bolaños Tinjaca \\ Johann Enrique Pirela Morillo \\ Universidad de La Salle, Colombia
}

CASE REPORT

\begin{abstract}
Resumen
Objetivo. Proponer un portafolio de servicios y actividades aplicables en las bibliotecas de la Universidad de La Salle (Bogotá-Colombia), con objeto de contribuir con la construcción de la paz, a partir de considerar la biblioteca universitaria como un laboratorio, generador de servicios y productos alineados con las agendas de desarrollo socio-cultural y educativo de la sociedad colombiana afectada por el conflicto armado del país.

Método. Enfoque mixto, sustentado en la entrevista y la encuesta como técnicas de investigación. Las entrevistas se realizaron a directivos de la Universidad, explorando sus concepciones acerca de cómo las Bibliotecas de la institución pueden apoyar los procesos de construcción de la paz, mediante servicios y actividades. La encuesta se aplicó, mediante un cuestionario estructurado, dirigido a estudiantes cursantes de la cátedra lasallista, asignatura que se estudia en todos los programas académicos y facultades, la cual imprime la impronta que distingue la filosofía que fundamenta la Universidad, uno de cuyos principios fundamentales es precisamente valores como la tolerancia, justicia, fe y paz .

Resultados. Luego de la triangulación de resultados, se propone el portafolio de servicios y actividades, com objeto de contribuir con el desarrollo de la cultura de paz desde la educación superior y com ello escalar hacia mejores condiciones de bienestar y equilibrio social.
\end{abstract}

Palabras clave

Bibliotecas universitarias; Colombia; construcción de la paz; educación para la paz; servicios y productos

\section{Proposal of a portfolio of services and activities for peacebuilding from the Libraries of the University of La Salle (Bogota, Colombia)}

\begin{abstract}
Objective. This article proposes a portfolio of services and activities applicable in the libraries of the University of La Salle (Bogotá-Colombia), in order to contribute to the construction of peace, considering the university library as a laboratory, generator of services and products aligned with the agendas of socio-cultural and educational development of Colombian society affected by the armed conflict of the country.

Method. Mixed approach, based on the interview and the survey as research techniques. The interviews were conducted with executives of the University, exploring their conceptions about how the Libraries of the institution can support the processes of peace building, through services and activities. The survey was applied, through a structured questionnaire, aimed at students of the Lasallian Chair, a subject that is studied in all academic programs and faculties, which prints the imprint that distinguishes the philosophy underlying the University, one of whose fundamental principles It is precisely values such as tolerance, justice, faith and peace.

Results. After the triangulation of results, the portfolio of services and activities is proposed, with the aim of contributing to the development of the culure of peace from higher education and as a way to scale towards better conditions of well-being and social equilibrium.
\end{abstract}

Keywords

Colombia; peace building; peace education; peace education; services and products; university libraries 


\section{Introducción}

Las Bibliotecas de la Universidad de La Salle consideran como parte de sus objetivos misionales actuar como punto de encuentro para el acceso a la información y al desarrollo de actividades académicas e investigativas, proporcionando una gran variedad de recursos bibliográficos y audiovisuales, así como actividades especiales para los jóvenes, docentes y personal administrativo. Servicios que siempre están a disposición de todos los usuarios sin costo alguno, satisfaciendo las necesidades de información y contribuyendo con el aprendizaje permanente, lo cual a su vez permite la formación integral de los profesionales, sin tener en cuenta edad, sexo, religión, nacionalidad, idioma o condición social. Igualmente, la Universidad de La Salle en su Proyecto Educativo Universitario (PEUL, 2007) establece como misión el fomento de una educación integral y la generación de conocimiento que aporte a la transformación social y productiva del país.

Con base en estos fundamentos, las Bibliotecas de la Universidad deben ser el escenario fundamental para apoyar la formación de ciudadanos y ciudadanas, promotores de la democracia y el progreso integral de la sociedad, ofreciendo aspectos claves en el desarrollo de seres humanos capaces de propiciar el diálogo, el respeto y la tolerancia. Se busca entonces, que las Bibliotecas de la Universidad de La Salle, desde su contexto social y educativo, contribuyan con la construcción de la paz en Bogotá y en Colombia.

La propuesta de servicios y de actividades que se formulan para las Bibliotecas de la Universidad de La Salle surgió como resultado de una investigación que se planteó como objetivo abordar un tema de gran importancia en la actualidad a nivel mundial: la Educación para la Paz y las estrategias para crear una cultura de Paz desde las bibliotecas en las instituciones de educación superior, lo cual se revertirá también a los demás estamentos de la sociedad, si se asume como parte esencial en la configuración de los proyectos éticos de vida. La construcción de la paz se justifica en estos momentos debido a que las diferencias y los conflictos entre los seres humanos, en su mayor parte, son resueltos utilizando la violencia, problemática que ha incitado alcanzar una conciencia sobre la importancia de preservar la paz, como un mecanismo además de cohesión social.

En este sentido, fue importante conocer los procesos y las actividades que se desarrollan en las instituciones de educación superior, reconociendo el potencial de construcción de actitudes positivas hacia una paz genuina y duradera, en la comunidad estudiantil (futuros dirigentes del país). Por tal motivo, se consideró pertinente proponer un portafolio de actividades, servicios y productos para la Biblioteca de La Universidad de La Salle orientado hacia la construcción de la paz, mediante la puesta en marcha estrategias efectivas para la comunidad universitaria.

\section{Fundamentación teórica}

La construcción de la paz siempre ha estado vinculada con los procesos formativos y con el conjunto de actividades y apuestas que las instituciones educativas deben incluir como parte de sus planes de estudios y sus estrategias para garantizar una educación cada vez más cimentada en el fomento de valores y principios. En el caso particular de las instituciones de educación superior estas acciones pueden ayudar a sentar las bases de un futuro sostenible y una de las formas de lograrlo es diseñando y aplicando mecanismos que contribuyan con la formación integral de los profesionales, lo cual no solo alude al desarrollo de competencias técnicas o el dominio de conocimientos y destrezas para desempeñarse eficazmente en un área de trabajo, sino que el propósito que las universidades deben buscar en todo momento es una preparación altamente calificada de cuadros profesionales, considerando las dimensiones éticas y morales, dentro de las cuales se incluye el cultivo de principios compatibles con la construcción de escenarios de paz y tolerancia.

Según UNESCO (2009), la Educación Superior debe no sólo proporcionar competencias sólidas para el mundo de hoy y de mañana, sino contribuir además a la formación de ciudadanos dotados de principios éticos, comprometidos con la construcción de la paz, lo cual a su vez no se determina únicamente por la difusión de la información sobre la necesidad de crear ambientes de paz, sino que es preciso tener en cuenta actividades educativas basadas en la tolerancia, el respeto, generando espacios innovadores y alternativos basados en aspectos científicos, tecnológicos, educativos y de participación, permitiendo la comprensión de un entorno social de reflexión compartida en la que se fomente una Cultura de Paz.

En Colombia, no se desconoce que, durante muchos años, los ciudadanos han estado sometidos a la violencia y a la guerra. Se trata de un conflicto, para cuya solución deben comprometerse los actores y escenarios educativos, políticos, económicos, sociales y culturales. Cada vez más, las estadísticas de mortalidad de seres humanos inocentes aumentan sin control alguno, producto no solo de acciones bélicas sino también de evidentes demostraciones de intolerancia. De acuerdo con lo anterior, cabe resaltar que los líderes en Colombia han manifestado de diferentes formas, la búsqueda de soluciones a esta problemática de forma pacífica. Para 
Gualy (2015), el ejemplo más próximo de la búsqueda de la paz en el país ha sido la negociación como salida al conflicto armado con las 30 Fuerzas Armadas Revolucionarias- Ejército del Pueblo (FARC-EP).

Puede observase el interés de instituciones gubernamentales tanto públicas como privadas por alcanzar la paz, buscando diferentes alternativas de forma innovadora para la construcción de una Cultura de Paz. Por lo tanto, es importante investigar si la sociedad especialmente las instituciones de Educación Superior se encuentran preparadas para la construcción de la Paz y cómo pueden contribuir desde sus funciones de docencia, investigación y extensión.

Según Echevarría (2010), los objetivos de la pedagogía para la paz deben responder a propósitos variados. Por un lado, parten de reconocer en los orígenes de la violencia y el conflicto producido en las relaciones cotidianas que día a día enfrentan los ciudadanos. Frente a la necesidad de recuperación de la paz y del equilibrio social, se requiere entonces instaurar una pedagogía para la paz desde las universidades a partir de elementos, actividades y procesos que apuesten por a la no violencia y la solución de conflictos, reconociendo el papel preponderante de accione de mediación, como estrategias que fomentan el cultivo de la convivencia pacífica.

En esta misma línea, se habla también de una educación para la paz, la cual en términos de Mayor (2014), debe enseñar no solo los beneficios de la concordia y del entendimiento sino a desaprender la violencia, a desprogramar conductas de predominio e intolerancia, generadoras de exclusión, desigualdad, discriminación, pobreza, injusticia, intimidación entre otros infortunios para las personas. La educación para la paz facilita la creación de espacios de encuentro apoyados en el diálogo, el respeto a las diferentes formas de pensar, el reconocimiento, la reflexión, la convivencia, la aceptación y la solución de conflictos, contribuyendo a despertar una conciencia humana, previniendo conflictos, generando una disposición pacifica, buscando opciones a través de concesos para la solución de problemáticas que afectan a la sociedad en todos sus ámbitos de la vida personal, familiar y profesional, por lo tanto es de gran importancia adquirir desde la universidad una formación integral en la que se logren adquirir hábitos, destrezas y métodos que permitan a las personas desarrollarse íntegramente.

Cabe destacar que en el Proyecto Educativo Universitario Lasallista (PEUL), se menciona un compromiso fundamental: "Educar para Pensar, Decidir y Servir" y este compromiso brinda a las generaciones que encuentran en esta propuesta, la posibilidad de construir una sociedad pluralista, respetuosa de los Derechos Humanos, orgullosa de los elementos propios de la cultura nacional y de sus tradiciones, se inserta en un mundo globalizado que espera transformaciones hacia la justicia y el desarrollo integral y sustentable." (PEUL, 2007). Desde este contexto, la Universidad de La Salle se esmera no solo para formar profesionales con sentido propio, sino con carácter humano, ético y moral, convirtiéndolos en buenos ciudadanos capaces de aportar a la sociedad con calidad.

En este sentido, entendemos que la Biblioteca Universitaria más que un instrumento de apoyo a las funciones de las instituciones de educación superior, debe ser un órgano de importancia estratégica, articulado con el currículo, en tanto que proyecto histórico, pedagógico y organizacional, que define la utopía social a la cual se debe aspirar, explorando alternativas activas, a partir de las cuales, la comunidad académica encuentre ofertas de productos y servicios de información, que no solo se caractericen por ser innovadores, sino que apunten on el fin de contribuir en la promoción de valores éticos, alcanzando un desarrollo social equitativo y sostenible en ambientes de paz en la formación de buenos ciudadanos.

De acuerdo con las Directrices de III Plan Estratégico de Rebium 2020, (Red Española de Bibliotecas Universitarias) y la Comisión Sectorial de CRUE (Conferencia de Rectore de Universidades Españolas), en una de las líneas estratégicas se proponen como uno de sus objetivos generales: "Promover la integración de la biblioteca en los objetivos de la universidad. Fortalecer alianzas con otros servicios universitarios e implicar a las bibliotecas universitarias en el ámbito de la responsabilidad social de la universidad." (REBIUM, 2016).

Con el desarrollo del propósito anteriormente mencionado se obtendría un desempeño importante en la creación y ejecución de actividades culturales encaminadas a la construcción de la paz, convirtiéndose en escenarios ideales que propicien comunicación, participación y convivencia universitaria sana, a través del ofrecimiento del conocimiento y a la educación permanente, ya que es un derecho fundamental de los seres humanos.

De esta forma, la Biblioteca de la Universidad de La Salle ha de establecer servicios, actividades culturales atractivas que atraigan a la comunidad universitaria en cada una de las sedes, ya que solo de esta forma se garantiza que se cumpla con uno de los proyectos que ha precisado la Biblioteca de la Universidad de La Salle en el PID (Plan Institucional de Desarrollo, 2015-2020) donde se define los proyectos y metas de los programas estratégicos para los próximos cinco años. Con el Fomento de la lectura y la divulgación cultural con la meta de 
"Consolidar la agenda cultural de la biblioteca" PID (Plan Institucional de Desarrollo, 2015-2020), desde esta iniciativa la universidad se proyectaría social y culturalmente, donde se inculque los valores como el respeto, el diálogo intercultural, la dignidad humana, la libertad de expresión, la tolerancia, la convivencia y la solidaridad.

Sobre la base de los argumentos desarrollados, las Bibliotecas de la Universidad de La Salle, deben ser entes mediadores de procesos formativos, que no solo facilite el acceso a la información tanto física como electrónica, sino en la formación de un espíritu crítico, reflexivo, con la capacidad de ser creativo y saber defenderse de las circunstancias que se presente en la vida cotidiana, facilitando una adecuada participación ciudadana en el conocimiento de sus deberes y derechos, desenvolviéndose en la sociedad por medio de la obtención de conocimientos sociales, científicos y tecnológicos, para lograr una mejor calidad de vida.

\section{Estrategia metodológica}

La estrategia metodológica concebida para llegar a la propuesta del portafolio de servicios y actividades que las Bibliotecas de la Universidad de La Salle pueden ofrecer, se estructuró con base en el enfoque mixto, que reconoce el potencial de la triangulación de técnicas e instrumentos de recolección de datos como alternativas para obtener una visión mucho más profunda de la realidad que se estudia, llegando a generar resultados que enriquecen las propuestas formuladas.

Las dos técnicas aplicadas en el estudio fueron la entrevista y la encuesta. La primera se orientó a explorar las concepciones de directivos de la Universidad, sobre la pertinencia de que las Bibliotecas ofrezcan servicios y actividades que fomenten el desarrollo de valores y principios compatibles con la construcción de la paz, mediante acciones concretas e intencionadas, articuladas a su vez con el Proyecto Educativo Universitario que orienta los ejercicios formativos en la Universidad. Se entrevistaron a cuatro actores claves de la Universidad de La Salle, que lideran acciones estratégicas, relacionadas con la definición y puesta en práctica de políticas curriculares orientadas a promover y desarrollar la cultura de paz en la Universidad. Tales actores fueron dos docentes del Departamento de Formación Lasallista, unidad académica responsable de administrar las asignaturas relacionadas con la formación ética, dentro de la cual se incluyen contenidos y estrategias sobre cultura de paz y otros valores ciudadanos. Se entrevistaron también a dos directivos, que formulan y coordinan las políticas académicas y curriculares. Las preguntas incluidas en el guion de entrevista estructurada fueron las siguientes: concepción de la cultura de paz, actividades desarrolladas desde las bibliotecas de la Universidad de La Salle para promover la cultura de paz, contribución de las bibliotecas a la enseñanza de los contenidos de las asignaturas de formación lasallista.

La segunda técnica consistió en una encuesta, apoyada en un cuestionario estructurado dirigido a noventa estudiantes de diferentes Programas Académicos de la Universidad y cursantes de la asignatura: Cátedra Lasallista, que administra el Departamento de Formación Lasallista. Cabe mencionar que esta asignatura hace parte de todo un componente curricular que imprime el sello distintivo, el cual traduce la filosofía en torno a la cual se estructura la Universidad. Las preguntas realizadas a los estudiantes estuvieron orientadas hacia los siguientes aspectos: contribución de la asignatura cátedra lasallista a la formación de valores de paz, valores concretos que se consideran se han desarrollado o promovido desde la clase de cátedra lasallista, actividades que las bibliotecas de la Universidad pueden ofrecer para fomentar la cultura de paz.

\section{Propuesta del portafolio de servicios y actividades para el fomento de la paz desde las Bibliotecas de la Universidad de La Salle}

Luego de triangular la información generada de la aplicación de las técnicas e instrumentos señalados, y de analizar dicha información a la luz de los planteamientos teóricos realizados, se propone un portafolio de actividades, servicios y productos para ser aplicados en las Bibliotecas de la Universidad de La Salle, encaminados a promover una Cultura de Paz, para toda la comunidad universitaria, lo que permitirá contribuir a los objetivos, misión y visión de la Institución. En el portafolio se hace patente la concepción de los directivos, docentes y estudiantes incluidos en el estudio, quienes coincidieron en asumir una cultura de paz como en conjunto de valores, tales como la tolerancia, el respeto, la solidaridad, el diálogo, la mediación, entre otros. La paz fue considerada por los entrevistados como la posibilidad concreta de convivir, en un mundo diverso y plural, reconociendo las diferencias como riqueza de la naturaleza humana. 
En esta propuesta de portafolio se integran un conjunto de actividades que permitirán revitalizar las Bibliotecas de la Universidad, incorporándolas de una forma mucho más orgánica e integrada con el currículo, de modo concreto con los contenidos y competencias que se desarrollan desde las asignaturas del componente de formación lasallista, con lo cual se contribuiría con la paz y al fortalecimiento el diseño de estrategias creativas e innovadoras, ya que como lo menciona Rementería (2002) citado por Sánchez \& Pérez (2014, p.101), la biblioteca universitaria además de desarrollar funciones específicas como agente comunicador, tiene roles sociales vinculados con la creación, mantenimiento y transmisión de la producción intelectual de las instituciones de educación superior.

El portafolio puede llegar a ser de gran aporte para la comunidad Lasallista, debido a que es una oportunidad para crear un proceso formativo de enseñanza-aprendizaje que permita vivenciar la paz, reconociéndose como seres humanos dignos capaces de crear acciones sanas e inteligentes ante las injusticias, las opresiones violentas y excluyentes en las que vive la ciudadanía.

Las actividades, servicios y productos propuestos son:

\subsection{Clubes de lectura}

Los clubes de lectura son espacios de encuentro para compartir lecturas programadas por un grupo de personas, es una práctica sociocultural donde se reflexiona, crítica, e interpreta o intercambia la impresión de dicho tema, esta actividad se encuentra encaminada a motivar, incentivar y fortalecer el gusto e interés por la lectura.

Para Arranz, J. (2013). "los clubes, las bibliotecas y las políticas de fomento de la lectura son estrategias que contribuyen a incidir en el cambio educativo, en la formación de personas con unos valores y con unos hábitos más democráticos, más positivos que los que están trasmitiendo a veces otras instancias" (p. 76), por lo tanto, es una canal que permite conformar relaciones humanas en las cuales se pueden desarrollar capacidades personales como inculcar un pensamiento reflexivo para actuar con libertad, autonomía y solidaridad, asumiendo responsabilidades que permitan aprender a convivir en armonía.

Realizar clubes de lectura enfocados en temas relacionados con la Paz es dar a conocer el contexto histórico y cultural por el que ha tenido que afrontar el país, es una actividad que permite crear una Cultura de Paz, a través de dialogo compartiendo percepciones, sentimientos y experiencias en común.

\begin{tabular}{|c|c|c|c|c|}
\hline Actividades & Objetivo & Indicadores de logro & Estrategias & $\begin{array}{c}\text { Valores que } \\
\text { contribuyen } \\
\text { con la paz }\end{array}$ \\
\hline $\begin{array}{l}\text { Clubes } \\
\text { de lectura }\end{array}$ & $\begin{array}{l}\text { Desarrollar } \\
\text { competencias } \\
\text { lectoras en las } \\
\text { que se utilicen } \\
\text { transversalmente } \\
\text { valores de } \\
\text { tolerancia, } \\
\text { respeto y } \\
\text { conciencia. }\end{array}$ & $\begin{array}{l}\text { - Libre expresión de los } \\
\text { participantes (sentimientos, } \\
\text { emociones, creencias). } \\
\text { - Conoce la realidad del } \\
\text { País. } \\
\text { - Conoce los derechos y } \\
\text { deberes de los ciudadanos. } \\
\text { - Genera empatía con los } \\
\text { miembros del mismo grupo } \\
\text { de trabajo. } \\
\text { - Participación colectiva. } \\
\text { - Desarrolla el pensamiento } \\
\text { crítico. } \\
\text { - Emplea el debate como } \\
\text { canal de comunicación. } \\
\text { - Valora la diversidad } \\
\text { ideológica como base del } \\
\text { respeto hacia los demás. }\end{array}$ & $\begin{array}{l}\text { Encuentro con escritores que desarrollen el } \\
\text { tema de la Paz. } \\
\text { Participación de autoridades, líderes } \\
\text { comunitarios. } \\
\text { Presentación de libros referidos al tema de } \\
\text { la Paz. } \\
\text { Organización de representaciones teatrales } \\
\text { o espectáculos con temas de Paz. } \\
\text { Organización de concursos de relatos o } \\
\text { dibujos inspirados en la lectura entre los } \\
\text { miembros del club. } \\
\text { Organización Café literario. } \\
\text { Lectura en voz alta (poemas, cuentos, } \\
\text { lecturas) }\end{array}$ & $\begin{array}{l}\text { Diálogo } \\
\text { Respeto } \\
\text { Tolerancia } \\
\text { Igualdad } \\
\text { Solidaridad } \\
\text { Responsabilidad } \\
\text { Reflexión } \\
\text { Sociabilidad }\end{array}$ \\
\hline
\end{tabular}




\subsection{Debates}

El debate es una estrategia, por medio de la cual se logra comunicar, plantear, conocer y exponer diferentes ideas sobre un tema específico, el cual se pone más interesante a medida que las posturas, aportes y argumentos sean puestos en la mesa, con la finalidad de llegar a una o varias conclusiones. Abordar temas relacionados con la Paz a través de actividades como los debates desarrolla en los seres humanos la capacidad de dialogar, comunicarse y obtener soluciones no violentas, propiciando el progreso de una consciencia de paz donde cada ciudadano fortalece valores, creencias y formas de relacionarse con las demás personas.

"Se requiere fomentar un diálogo afectivo, de verdades escucha del otro, en los temas fuente o foco de tensión, de conflicto, de altercado, se trata de, llegar al punto de los sujetos tengan la capacidad y predisposición al diálogo argumentado en la búsqueda de alternativas de solución" (Ceballos, 2013).

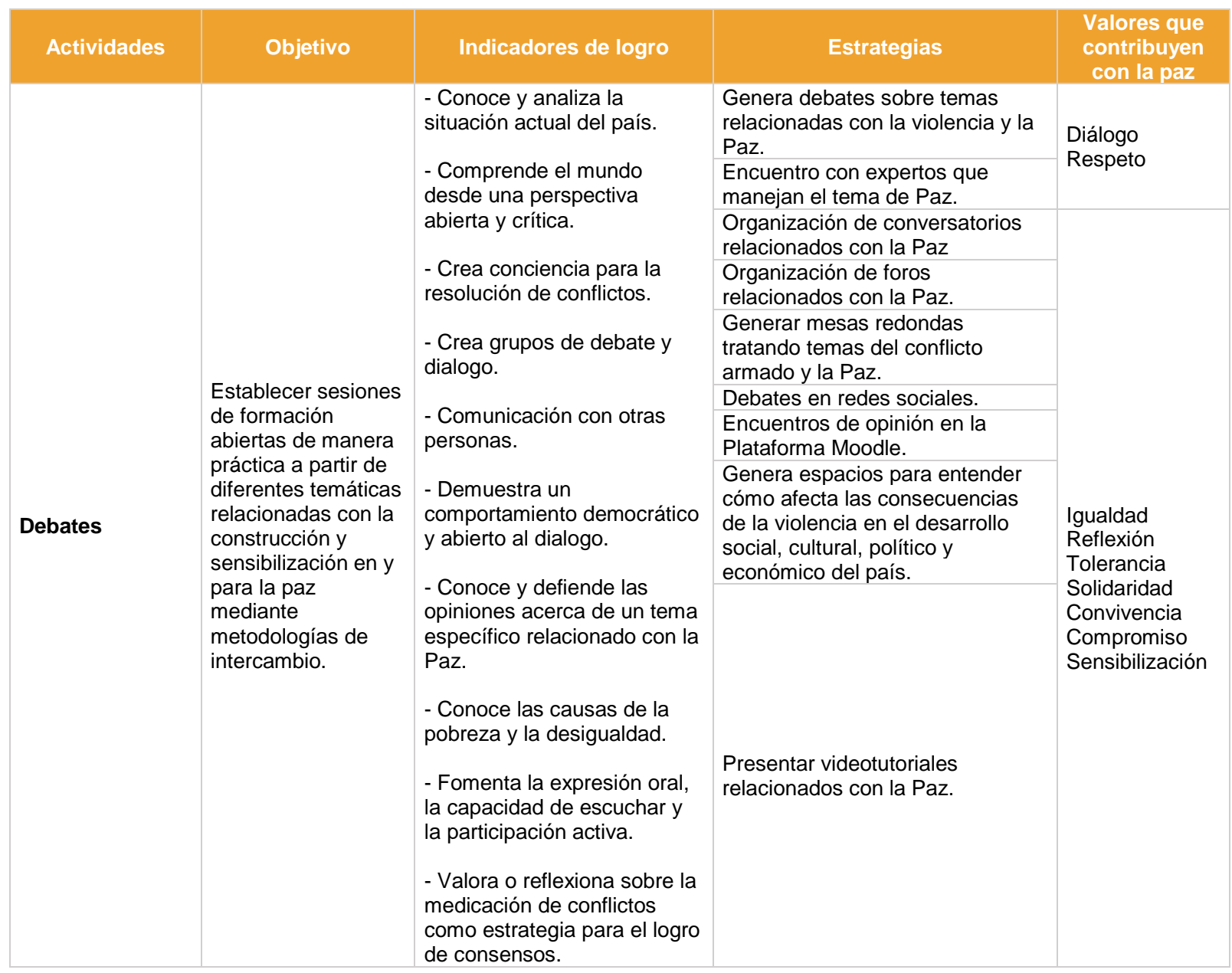

Cuadro 2 - Debates

Fuente: Elaboración propia.

\subsection{Exposiciones}

Una exposición es una actividad donde se realiza una exhibición o muestra de objetos, documentos u otros materiales con diferentes temáticas y variables, dirigida a un grupo específico de personas o al público en general, es una estrategia para mostrar la realidad, la violación de los derechos humanos, a partir de los hechos o acontecimientos presentados con el conflicto armado y el proceso de Paz por el que afronta el país.

Al respecto Cabezudo. (2012) opina que "la educación debe proveer la posibilidad de un desarrollo integral en el cual la adquisición de valores universales y el conocimiento de los derechos humanos colaboren para que los estudiantes se involucren como actores dinámicos y transformadores de la realidad" ( $p-142)$. 
Sensibilizar a la comunidad universitaria por medio de estas actividades permite mostrar la realidad, compartir experiencia y adquirir conocimiento, convirtiéndose en prácticas sociales con el objeto en la búsqueda de posibles soluciones a nivel individual o colectivo.

\begin{tabular}{|c|c|c|c|c|}
\hline Actividades & Objetivo & Indicadores de logro & Estrategias & $\begin{array}{l}\text { Valores que } \\
\text { contribuyen } \\
\text { con la paz }\end{array}$ \\
\hline \multirow{7}{*}{$\begin{array}{l}\text { Exposiciones } \\
\text { (Memoria } \\
\text { Histórica) }\end{array}$} & \multirow{7}{*}{$\begin{array}{l}\text { 1. Propiciar la } \\
\text { conformación de } \\
\text { espacios donde } \\
\text { profesionales, } \\
\text { profesores, egresados } \\
\text { y estudiantes, tengan } \\
\text { la oportunidad de } \\
\text { presentar trabajos de } \\
\text { calidad, generando } \\
\text { reflexiones a partir de } \\
\text { temas relacionados } \\
\text { con la Paz. } \\
\text { 2. Contribuir con } \\
\text { procesos de } \\
\text { democratización de la } \\
\text { memoria y en } \\
\text { particular a procesos } \\
\text { de verdad, justicia, } \\
\text { reparación y no } \\
\text { repetición de hechos } \\
\text { no violentos. }\end{array}$} & \multirow{7}{*}{ 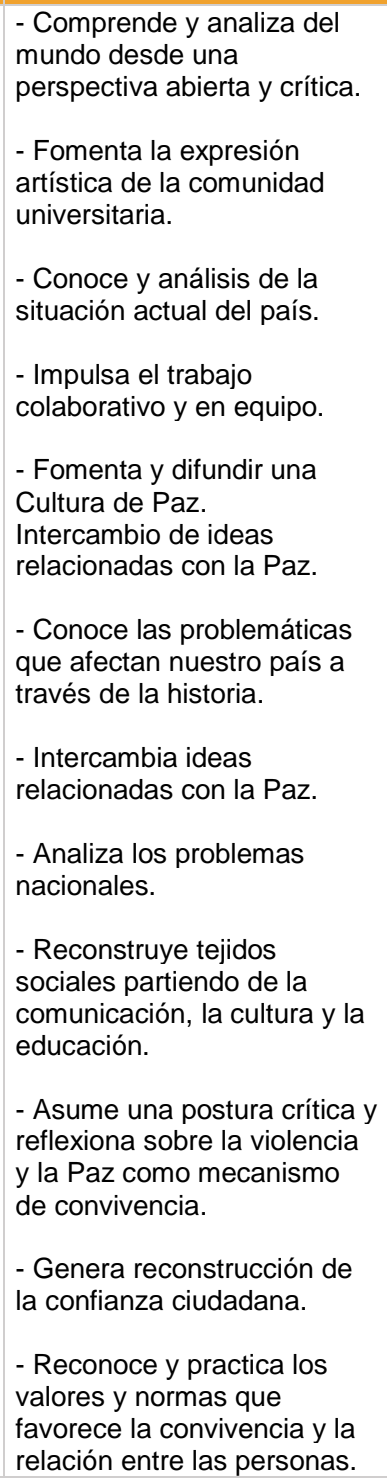 } & $\begin{array}{l}\text { Exposición fotográfica } \\
\text { relacionada con } \\
\text { investigaciones sobre temas } \\
\text { específicos vinculados con el } \\
\text { conflicto armado. } \\
\text { Exposición de documentales } \\
\text { que muestren la violencia } \\
\text { que han vivido y que siguen } \\
\text { viviendo las comunidades } \\
\text { campesinas, negras e } \\
\text { indígenas desde tiempo } \\
\text { atrás. }\end{array}$ & \multirow{7}{*}{$\begin{array}{l}\text { Reflexión } \\
\text { Sensibilización } \\
\text { Socialización } \\
\text { Respeto } \\
\text { Reflexión } \\
\text { Humildad } \\
\text { Trabajo en } \\
\text { equipo } \\
\text { Solidaridad }\end{array}$} \\
\hline & & & $\begin{array}{l}\text { Exposiciones audiovisuales } \\
\text { (audios, videos y testimonios) } \\
\text { que den a conocer a los } \\
\text { usuarios el contexto sobre las } \\
\text { masacres de hechos } \\
\text { ocurridos en nuestro país. }\end{array}$ & \\
\hline & & & $\begin{array}{l}\text { Exposición de resultados de } \\
\text { Investigaciones realizadas } \\
\text { por partes de los docentes de } \\
\text { la Universidad relacionados } \\
\text { con temas de construcción } \\
\text { de Paz. }\end{array}$ & \\
\hline & & & $\begin{array}{l}\text { Exposición de libros } \\
\text { relacionado con tema de la } \\
\text { Paz. }\end{array}$ & \\
\hline & & & $\begin{array}{l}\text { Exposición de Revistas } \\
\text { relacionadas con temas de la } \\
\text { Paz. }\end{array}$ & \\
\hline & & & $\begin{array}{l}\text { Exposición de temas } \\
\text { relacionados con la paz en } \\
\text { medios digitales y } \\
\text { electrónicos. }\end{array}$ & \\
\hline & & & $\begin{array}{l}\text { Exposición de Pintura y Arte } \\
\text { con temas relacionados con } \\
\text { la Paz. }\end{array}$ & \\
\hline
\end{tabular}




\subsection{Videoconferencias y cine-foros}

La videoconferencia es una actividad que permite una comunicación de manera simultánea donde se combina el audio y el vídeo, con esto se logra obtener reuniones con grupos de personas ubicadas en cualquier lugar del mundo para interactuar en temas específicos.

Rendón (2015) opina que la "videoconferencia es sumergir a la ciudadanía digital en un océano de nuevas posibilidades formativas basadas en la creación de conocimiento compartido, en la resolución colaborativa de problemas y en la búsqueda de nuevos horizontes personales y sociales" ( $p-55)$. Por lo tanto, es una actividad que contribuye con una Cultura de Paz, ya que a través de esta se pueden contactar personajes que tratan temas de Paz y así mismo contextualizar a la comunidad Universitaria por medio de la interacción con los demás actores de la sociedad a nivel internacional.

\begin{tabular}{|c|c|c|c|c|}
\hline Actividades & Objetivo & Indicadores de logro & Estrategias & $\begin{array}{l}\text { Valores que } \\
\text { contribuyen } \\
\text { con la paz }\end{array}$ \\
\hline \multirow{6}{*}{$\begin{array}{l}\text { Video conferencias } \\
\text { y cineforos }\end{array}$} & \multirow{6}{*}{$\begin{array}{l}\text { Impulsar el } \\
\text { desarrollo de } \\
\text { actividades de } \\
\text { socialización del } \\
\text { conocimiento } \\
\text { relacionadas con la } \\
\text { paz sustentada en } \\
\text { medios digitales y } \\
\text { audiovisuales. }\end{array}$} & \multirow{2}{*}{$\begin{array}{l}\text { - Compartir ideas, } \\
\text { conocimientos, } \\
\text { información. } \\
\text { - Planear estrategias } \\
\text { dirigidas a la solución de } \\
\text { conflictos en su ámbito } \\
\text { de influencia. } \\
\text { - Crea comunicación con } \\
\text { investigadores en temas } \\
\text { de Paz. }\end{array}$} & $\begin{array}{l}\text { Conferencias con temas } \\
\text { especializados de Paz. } \\
\text { Lograr convenios con } \\
\text { instituciones como RENATA que } \\
\text { facilite actividades científicas, } \\
\text { educativas y de innovación. } \\
\text { Ofrecimiento de } \\
\text { videoconferencias a nivel grupal o } \\
\text { individual. }\end{array}$ & $\begin{array}{l}\text { Sensibilización } \\
\text { Cooperación } \\
\text { Reconciliación y } \\
\text { perdón. }\end{array}$ \\
\hline & & & $\begin{array}{l}\text { Interconexión con entes } \\
\text { gubernamentales que trabajen } \\
\text { con la Paz. }\end{array}$ & Convivencia \\
\hline & & \multirow{4}{*}{$\begin{array}{l}\text { - Intercambia ideas } \\
\text { relacionadas con la Paz. } \\
\text { - Aborda situaciones de } \\
\text { conflicto y mal trato. } \\
\text { - Contextualización de } \\
\text { los avances y la } \\
\text { planificación del } \\
\text { abordaje de los temas } \\
\text { relacionados con la Paz. } \\
\text { - Reflexiona sobre el } \\
\text { impacto de las } \\
\text { situaciones de violencia } \\
\text { como un elemento que } \\
\text { genera desequilibrio } \\
\text { social. }\end{array}$} & $\begin{array}{l}\text { Interconexión con entes } \\
\text { internacionales que se interesen } \\
\text { por la Paz. }\end{array}$ & \multirow[b]{4}{*}{$\begin{array}{l}\text { Respeto } \\
\text { Comprensión, } \\
\text { generosidad } \\
\text { Igualdad } \\
\text { Integridad }\end{array}$} \\
\hline & & & $\begin{array}{l}\text { Organizar cine foros relacionados } \\
\text { con la Paz. }\end{array}$ & \\
\hline & & & $\begin{array}{l}\text { Proyecta películas especializadas } \\
\text { en temas de la Paz. }\end{array}$ & \\
\hline & & & $\begin{array}{l}\text { Documentales publicados en } \\
\text { diferentes medios de } \\
\text { comunicación. }\end{array}$ & \\
\hline
\end{tabular}

Cuadro 4 - Video conferencias y cineforos

Fuente: Elaboración propia.

\subsection{Talleres}

Los talleres son actividades estratégicas que a partir de la práctica se adquiere conocimiento y formación personal, obteniendo resultado de un trabajo en equipo o colectivo donde se aprende a construir reglas, solucionar dificultades y reflexionar sobre el trabajo realizado.

Así mismo la realización de talleres como estrategia de Educación para la Paz es según Gómez, (2014) "una vivencia por la cual el individuo encuentra desafíos y dificultades. También aprende a encontrarse con las necesidades de los demás y a comprender mejor sus relaciones interpersonales; se muestra más satisfecho consigo mismo y con los otros" ( $p-178)$, de acuerdo con las autoras son espacios de respeto, tolerancia, de participación, generación de dialogo y convivencia. 


\begin{tabular}{|c|c|c|c|c|}
\hline Actividades & Objetivo & Indicadores de logro & Estrategias & $\begin{array}{l}\text { Valores que } \\
\text { contribuyen } \\
\text { con la paz }\end{array}$ \\
\hline Talleres & $\begin{array}{l}\text { Proporcionar a la } \\
\text { comunidad } \\
\text { universitaria un } \\
\text { repertorio de } \\
\text { posibilidades de } \\
\text { aprendizaje } \\
\text { cooperativo para el } \\
\text { desarrollar } \\
\text { habilidades } \\
\text { interpersonales como } \\
\text { comunicación, } \\
\text { empatía, trabajo en } \\
\text { equipo y solidaridad. }\end{array}$ & $\begin{array}{l}\text { - Valora sus acciones y las s } \\
\text { de los demás de la forma más } \\
\text { adecuada en una perspectiva } \\
\text { de violencia. } \\
\text { - Identifica características } \\
\text { positivas y negativas de } \\
\text { nosotros mismos y de las } \\
\text { personas que nos rodean. } \\
\text { - Interactúa con otras } \\
\text { personas. } \\
\text { - Integra y acepta a otras } \\
\text { personas distintas a las del } \\
\text { entorno habitual. } \\
\text { - Aporta al cambio social. } \\
\text { - Crea conciencia, } \\
\text { compromiso y } \\
\text { responsabilidad. } \\
\text { - Alcanza transformación } \\
\text { en procesos de aprendizaje. } \\
\text { - Aplica habilidades para } \\
\text { mantener y mejorar el clima } \\
\text { de convivencia. } \\
\text { - Comprende el derecho y } \\
\text { deber a la paz. } \\
\text { - Comprender las diferentes } \\
\text { formas de responsabilidad. }\end{array}$ & $\begin{array}{l}\text { Talleres relacionados con la Paz } \\
\text { y no violencia. } \\
\text { Talleres de estudios de caso } \\
\text { relacionados con la Paz. } \\
\text { Talleres de música y cine que } \\
\text { contribuyan con la Paz. } \\
\text { Talleres de escritura } \\
\text { Talleres de cómic } \\
\text { Taller cuidado del medio } \\
\text { ambiente. } \\
\text { Taller de Coaching Personal. } \\
\text { Taller de búsqueda de empleo } \\
\text { Taller de Autoestima, } \\
\text { Crecimiento Personal. } \\
\text { Taller sobre el contexto legal } \\
\text { relacionado con Derechos } \\
\text { Humanos y la Paz. } \\
\text { Talleres solo para docentes } \\
\text { relacionados con la Educación } \\
\text { para la Paz }\end{array}$ & $\begin{array}{l}\text { Trabajo en } \\
\text { equipo } \\
\text { Dignidad } \\
\text { Generosidad } \\
\text { Responsabilidad } \\
\text { Reflexión } \\
\text { Integridad } \\
\text { lgualdad } \\
\text { Respeto } \\
\text { Tolerancia } \\
\text { Convivencia } \\
\text { Cooperación } \\
\text { Solidaridad }\end{array}$ \\
\hline
\end{tabular}

\subsection{Diseño de productos de información}

El diseño de productos informativos es una oportunidad para ofrecer a la comunidad universitaria, información confiable, es una estrategia clave para orientar a los ciudadanos a una Cultura de Paz, de dar a conocer los derechos y deberes que tienen cada ciudadano, expresar sus ideas libremente, manifestar o denunciar y así alcanzar igualdad y democracia para todos. Para una acertada y correcta difusión de la información, según Martínez, O \& Burgos, P. (2014) "se requiere de un enfoque de ciudadanías comunicativas que tomen en cuenta lo territorial, lo cultural y lo sociopolítico como dinamizadores transversales de estrategias informativas y comunicativas orientadas a superar el conflicto armado y viabilizar la construcción de paz" (p-45).

\begin{tabular}{|c|c|c|c|c|}
\hline Actividades & Objetivo & $\begin{array}{c}\text { Indicadores } \\
\text { de logro }\end{array}$ & Estrategias & $\begin{array}{l}\text { Valores que } \\
\text { contribuyen } \\
\text { con la paz }\end{array}$ \\
\hline \multirow{2}{*}{$\begin{array}{l}\text { Diseño de } \\
\text { productos de } \\
\text { información } \\
\text { para el } \\
\text { fomento de } \\
\text { la paz }\end{array}$} & \multirow{2}{*}{$\begin{array}{l}1 \text { - Diseñas productos (repositorios, } \\
\text { compilaciones digitales, sitios web, } \\
\text { portales blog. wikis) orientados hacia el } \\
\text { afianciamiento de valores de Paz. } \\
2 \text { - Generar una Red de apoyo entre la } \\
\text { Biblioteca, las Unidades Académicas y } \\
\text { los estudiantes. }\end{array}$} & \multirow{2}{*}{$\begin{array}{l}\text { - Indaga sobre } \\
\text { las fuentes } \\
\text { documentales } \\
\text { referidas a la } \\
\text { paz. } \\
\text { - Sistematiza } \\
\text { información } \\
\text { sobre las ideas } \\
\text { de paz. }\end{array}$} & $\begin{array}{l}\text { Elaboración de una cartera } \\
\text { informativa } \\
\text { Creación de un link en la página } \\
\text { WEB }\end{array}$ & \multirow{2}{*}{$\begin{array}{l}\text { Cooperación } \\
\text { Solidaridad } \\
\text { Responsabilidad }\end{array}$} \\
\hline & & & $\begin{array}{l}\text { Creación de una Base de Datos } \\
\text { electrónica creada y alimentada } \\
\text { por la Biblioteca. }\end{array}$ & \\
\hline
\end{tabular}




\section{Conclusiones}

En el ámbito de la educación superior el tema de la paz debe ser incluido no solo en las propuestas curriculares, bajo la figura de contenidos transversales y también como experiencias de formación que fomenten ambientes para el desarrollo de valores éticos como la tolerancia, el respeto al a diversidad y la reflexión acerca del impacto de las acciones humanas en los procesos de construcción del tejido social. Estos mismos valores y principios deben estar también presentes en la oferta de servicios, actividades y productos que las bibliotecas universitarias deben ofrecer para estar en sintonía con las demandas de las sociedades globales que también requieren globalizar la esperanza en un presente y futuro mejor.

Los resultados de la investigación realizada a directivos, docentes y estudiantes de la Universidad de La Salle ponen de relieve la necesidad de concretar acciones para promover la cultura paz, en un ambiente de fomento a la formación ciudadana. Las concepciones expresadas por los actores y actoras incluidas en el estudio, así como también las actividades sugeridas, como producto de las entrevistas y encuestas, enfatizaron en el hecho de que las Bibliotecas Universitarias deben operar en torno a estrategias educativas, que vayan mucho más allá de facilitar el acceso a la información tanto física como electrónica. En este sentido, las Bibliotecas de la Universidad de La Salle, enmarcadas en las premisas del Proyecto Educativo Universitario (PEUL), deben potenciar su dimensión mediadora y pedagógica, asumiéndose como un importante recurso de apoyo estratégico a la formador de profesionales con espíritu crítico, reflexivo, con la capacidad de ser creativo y saber cuándo defenderse de las circunstancias que se presente en la vida cotidiana, facilitando una adecuada participación ciudadana en el conocimiento de sus deberes y derechos, desenvolviéndose en la sociedad por medio de la obtención de conocimientos sociales, científicos y tecnológicos, para lograr una mejor calidad de vida.

Establecer una Cultura de Paz dentro de las actividades, servicios o funciones de la Biblioteca de La Universidad de La Salle, se direcciona en ejecutar sus actividades en tres áreas específicas: la cultural, la educativa y lo social, ya que de acuerdo al Enfoque Formativo Lasallista estos tres elementos hacen parte de un proceso "orientados a crear espacios adecuados para el acuerdo de normas y valores que favorezcan la convivencia, la tolerancia y la construcción de una ciudadanía democrática.

El portafolio integra los resultados del recorrido metodológico seguido, en el cual se consideró la visión de directivos, docentes y estudiantes, cuya información aportada fue muy valiosa para perfilar la propuesta. El éxito en la aplicación del portafolio propuesto implicará una sinergia entre directivos de la Universidad, el personal de difusión cultural de las Bibliotecas, docentes y estudiantes, logrando conformar una comunidad de aprendizaje sobre valores tan cruciales en estos momentos como la paz. Dicha comunidad debe trabajar de forma mancomunada, reconociendo su compromiso. La paz debe hacer parte de las estrategias que permean todas las asignaturas de los diferentes Programas Académicos de la Universidad y la Biblioteca debe estar ahí para reinventarse y convertirse en un laboratorio en el que se viva la paz, como garantía de contribuir con un futuro cada vez más sostenible.

\section{Referencias}

Arranz, J. (2013). Una relación significativa: los clubes de lectura y las redes de bibliotecas en Medellín y Barcelona. Revista Interamericana de Bibliotecología. 36, (1), 75-79. Recuperado de http://www.redalyc.org/pdf/1790/179029139007.pdf

Cabezudo, A. (2012). Educación para la paz: una construcción de la memoria, la verdad y la justicia. Desafío pedagógico de nuestro tiempo en América Latina. Revista Ciências Sociais Unisinos. 48, (2), 139-145. Universidade do Vale do Rio dos Sinos São Leopoldo, Brasil.

Ceballos, P. (2013). Educación para la paz y para la democracia. Revista UAIM, 9 (1), 35-48. Recuperado de: http://www.redalyc.org/html/461/46126366008/

\footnotetext{
Echevarría, C., (2010). Pedagogía para la paz en tiempos contemporáneos. Cátedra Lasallista 2009. Miradas sobre la reconciliación: reflexiones y experiencias. Colombia. Ediciones Unisalle.
}

Gómez, D. (2014) Paz en los colegios... ¿De dónde nos agarramos? Universidad Nacional de Colombia. Recuperado de: http://www.bivipas.unal.edu.co/bitstream/123456789/703/1/PAZ\%20EN\%20LOS\%20COLEGIOS\%E2\%80\%A6\%20DE\%20D\% C3\%93NDE\%20NOS\%20AGARRAMOS.pdf

Gulay, L., (2015) Construcción Curricular de una Cultura de Paz en América Latina Caso: Maestrías de Ciencias Políticas en Bogotá. (Trabajo de Grado, Universidad Nacional de Colombia). 
Martínez, Ó. y Burgos, P. (2015). Ciudadanías comunicativas y construcción de paz: la Agenda de Paz de Nariño Signo y Pensamiento. XXXIII, (65), 32-47. Pontificia Universidad Javeriana. Bogotá, Colombia.

Mayor, F. (2014). Educar a ser libres y responsables. Fundación cultura de paz. Revista Mediterráneos Económicos. 26.40.

Red Española de Bibliotecas Universitarias (REBIUN) (2016). III Plan estratégico de REBIUN. Recuperado de: http://www.rebiun.org/queesrebiun/Documents/III Plan\%20Estrategico REBIUN.pdf

Rendón, O (2015). Aprendizaje colaborativo mediante redes sociales y radio comunitaria web 2.0. Revista Lasallista de Investigación. 12, (2), pp. 54-65. Corporación Universitaria Lasallista. Antioquia, Colombia.

Sánchez, G., y Pérez, J., (2014) Gestión del conocimiento en bibliotecas universitarias: estrategias de creatividad e innovación Biblioteca Universitaria. Revista Biblioteca Universitaria. 17, (2). p. 103. México. Recuperado de http://www.redalyc.org/pdf/285/28540279002.pdf

UNESCO (2009). Conferencia Mundial sobre la Educación Superior - 2009: La nueva dinámica de la educación superior y la investigación para el cambio social y el desarrollo (Sede de la UNESCO, París, 5-8 de julio de 2009). Recuperado de: http://www.unesco.org/education/WCHE2009/comunicado es.pdf

Universidad de La Salle. (PEUL) (2007). Proyecto Educativo Universitario. Bogotá: Ediciones Unisalle. Librillos institucionales. Universidad de La Salle. (2015). Plan institucional de desarrollo. (2015-2020). Bogotá: Ediciones Unisalle. Librillos institucionales.

\section{Dados dos autores}

Martha Patricia Bolaños Tinjaca

Profesional en Sistemas de Información, Bibliotecología y Archivística por la Universidad de La Salle. Bogotá-

Colombia.

mpbolanos@lasalle.edu.co

Johann Enrique Pirela Morillo

Licenciado em Bibliotecología y Archivología. Magíster Scientiarum em Museología, Magíster em Educación. Mención: Currículo, Doctorado y Postdoctorado em Ciencias Humanas. Docente e investigador del Programa em Sistemas de Información, Bibliotecología y Archivística. Universidad de La Salle- Bogotá-Colombia

iepirela@unisalle.edu.co

Recibido - Received: 2017-08-03

Aceptado - Accepted: 2018-01-12

\section{(c) $\overline{E Y}$}

This work is licensed under a Creative Commons Attribution 4.0

United States License.

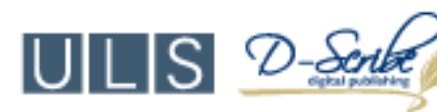

This journal is published by the University Library System of the University of Pittsburgh as part of its

D-Scribe Digital Publishing Program and is cosponsored by the University of Pittsburgh Press. 